Revue d'histoire de l'Amérique française

THEYUE D.HISTOIRE DE L'AMÉRIQUE FRANÇAISE

CENTRE DE RECHERCHE EN CIVILISATION

CANADIENNE-FRANÇAISE, UNIVERSITÉ D'OTTAWA, Actes du colloque sur la situation de la recherche sur la vie française en Ontario. Tenu à l'Université d'Ottawa les 28 et 29 novembre 1974. Organisé par le Centre de recherche en civilisation canadienne-française de l'Université d'Ottawa. Compte rendu publié en collaboration avec l'Association canadienne-française pour l'avancement des sciences (ACFAS), 1975. 280 p. $\$ 5.00$

\title{
Gaétan Gervais
}

Volume 30, numéro 1, juin 1976

URI : https://id.erudit.org/iderudit/303514ar

DOI : https://doi.org/10.7202/303514ar

Aller au sommaire du numéro

Éditeur(s)

Institut d'histoire de l'Amérique française

ISSN

0035-2357 (imprimé)

1492-1383 (numérique)

Découvrir la revue

Citer ce compte rendu

Gervais, G. (1976). Compte rendu de [CENTRE DE RECHERCHE EN CIVILISATION CANADIENNE-FRANÇAISE, UNIVERSITÉ D'OTTAWA, Actes $d u$ colloque sur la situation de la recherche sur la vie française en Ontario. Tenu à l'Université d'Ottawa les 28 et 29 novembre 1974. Organisé par le Centre de recherche en civilisation canadienne-française de l'Université d'Ottawa.

Compte rendu publié en collaboration avec l'Association canadienne-française pour l'avancement des sciences (ACFAS), 1975. 280 p. \$5.00]. Revue d'histoire de l'Amérique française, 30(1), 110-113. https://doi.org/10.7202/303514ar services d'Érudit (y compris la reproduction) est assujettie à sa politique d'utilisation que vous pouvez consulter en ligne. 
CENTRE DE RECHERCHE EN CIVILISATION CANADIENNE-FRANÇAISE, Université D'OtTAWA, Actes du colloque sur la situation de la recherche sur la vie française en Ontario. Tenu à l'Université d'Ottawa les 28 et 29 novembre 1974. Organisé par le Centre de recherche en civilisation canadienne-française de l'Université d'Ottawa. Compte rendu publié en collaboration avec l'Association canadienne-française pour l'avancement des sciences (ACFAS), 1975. 280 p. $\$ 5.00$

Souvent dissimulée comme une chose presque honteuse, la vie francoontarienne sort peu à peu de l'ombre. On peut en voir une preuve certaine dans l'intérêt que le Centre de recherche en civilisation canadienne-française 
(CRCCF) de l'Université d'Ottawa porte à la vie franco-ontarienne'. Le Centre a patronné à la fin de 1974 une réunion de plusieurs chercheurs intéressés à l'Ontario français afin de faire le point sur la Situation de la recherche sur la vie française en Ontario. Les communications présentées lors de ce colloque (ainsi que les commentaires sur chacune) viennent d'être publiées dans un volume.

Après le bref avant-propos du directeur du Centre, Pierre Savard, suivent une douzaine de communications couvrant presque tous les domaines des sciences sociales et humaines. Au départ, nous trouvons le folkloriste G. Lemieux, ( «La documentation orale dans le Nouvel-Ontario») l'éditeur d'une imposante collection de folklore franco-ontarien ${ }^{2}$, qui explique comment et pourquoi il a consacré plus de vingt-cinq années à recueillir des chansons et des contes franco-ontariens. B. Fortin ( $«$ La documentation imprimée sur l'Ontario français ou: une littérature de combat»), qui vient de rendre, avec J.-P. Gaboury, un grand "service aux chercheurs par la publication d'une bibliographie critique sur l'Ontario français ${ }^{3}$, fait une analyse des imprimés franco-ontariens: on note d'une part la prédominance du secteur de l'éducation et la pauvreté du secteur des sciences sociales, et d'autre part la prépondérance de la littérature de combat sous la forme de brochures, de mémoires, de pamphlets, etc. Robert Choquette, l'auteur récent d'une très intéressante étude sur les relations anglo-françaises dans l'Église catholique ontarienne ${ }^{4}$, fait le point sur «L'histoire des Franco-Ontariens: bilan de la recherche»; il présente un bilan, propose une périodisation pour aborder cette histoire, signale l'absence d'études d'envergure et apporte des suggestions quant aux études à entreprendre. R. D'Costa discute ensuite de «La population franco-ontarienne: quelques aspects démographiques». Sur le plan des études politiques, J.-P. Gaboury ( «La vie politique de l'Ontario français») constate lui aussi la misère de nos connaissances sur l'Ontario français: il suggère deux approches, une première par l'étude du pouvoir, l'autre par le biais de la culture politique du groupe. L'aspect sociologique fait ensuite l'objet d'un examen par Danielle Juteau-Lee et Jean Lapointe ( «État de la recherche en sociologie sur la vie des FrancoOntariens»): les secteurs culturel, politique et démographique sont examinés, et on aperçoit l'hypertrophie des préoccupations culturelles, ce qu'expliquent les impératifs de la lutte pour la survivance. On note d'autre part la faiblesse des apports théoriques et l'absence de préoccupation pour les problèmes sociaux (la délinquance, l'alcoolisme, la pauvreté, etc.). Jacques

P. Savard, «Du Centre et de la recherche en civilisation canadienne-française», in Bulletin du CRCCF, IV (2): 1-4. Bellarmin.

La collection «Les vieux m'ont conté» en est à son sixième volume chez

3 B. Fortin et J.-P. Gaboury, Bibliographie analytique de l'Ontario français (Ottawa, Éditions de l'Université d'Ottawa, Cahiers du CRCCF, 1975), xii-236p.

4 Robert Choquette, Language and religion. A history of English-French conflict in Ontario (Ottawa, University of Ottawa Press, 1975), xiv-264p. 
Henry ( Nature et conditions de la recherche en science économique») explique que la science économique, surtout quand elle n'est pas de gauche, n'est pas toujours adaptée à l'étude des minorités. Lionel Desjarlais examine la «Contribution franco-ontarienne à la recherche en éducation», retenant pour analyse toute la littérature pédagogique produite en français en Ontario, qu'elle porte ou non sur les Franco-Ontariens. Raymond Lamérand présente ensuite une communication sur «Le français parlé en Ontario» pour conclure que, malgré le mal qu'on dira de la qualité du français parlé en Ontario, "on n'arrive pas à découvrir chez les Franco-Ontariens une langue autre que le français» (p. 208). Pierre Hurtubise se penche ensuite sur «La vie religieuse des Franco-Ontariens», chez qui la langue et la religion ont été souvent confondues, comme au Québec: «tirez le fil de la religion, et c'est tout le tissu... qui vient»(p. 212), écrit Hurtubise. Après un tour de la documentation disponible, il recommande certaines orientations à suivre, et les sources pouvant y servir. Faisant le bilan de ses expériences d'animatrice culturelle depuis 1969, Micheline St-Cyr ( Art et culture en Ontario») plaide, sur un ton vif, en faveur des centres culturels et s'attaque aux obstacles mis sur leur chemin: certains organismes en place, le Québec, et même la France. Enfin, Gérard Lemieux («Pour une idéologie à base d'incidences sociócuiltuirelles régioñales») cherche, cñ idcntifiant certaines variables socio-culturelles, à décrire les collectivités francoontariennes.

L'ouvrage que nous offre le Centre de recherche en civilisation canadienne-française sera d'une grande utilité pour tous ceux qui s'intéressent à la vie franco-ontarienne: d'abord parce qu'il fait un inventaire de nos connaissances (plus souvent, de nos ignorances) dans de nombreux domaines de la connaissance, et deuxièmement parce que les auteurs des communications ont souvent indiqué l'orientation souhaitable des recherches futures. Aussi, les bibliographies qu'on trouve, soit incorporées dans les textes, soit à la fin des chapitres, ne manqueront pas de bien servir ceux qui voudront approfondir certaines questions. Le texte se présente bien, mais certaines erreurs, de frappe ou de français, ont échappé à la vigilance des correcteurs d'épreuves.

Ce qui frappe le lecteur du livre, c'est à quel point le bilan des recherches est maigre: partout, on aligne des questions pour lesquelles, souvent, il n'existe pas même des éléments de réponse. Cette faiblesse de la documentation et la carence des recherches expliquent sans doute l'absence de débats de fonds: il n'existe pas d'interprétation générale susceptible de susciter des discussions sur l'ensemble de la vie franco-ontarienne. En ajoutant que nous pouvons compter sur les doigts d'une main les véritables spécialistes de l'Ontario français, on comprend le peu d'avancement obtenu jusqu'à ce jour: les deux universités «bilingues» de l'Ontario commencent à peine à s'intéresser à ces questions, et elles doivent porter une partie de la responsabilité pour la situation actuelle. Les spécialistes ne pourront produire les études, les monographies, les synthèses souhaitées que dans la me- 
sure où ils auront les appuis institutionnels nécessaires: c'est là l'importance du volume publié ici et de l'intérêt du CRCCF. Que des spécialistes venus de disciplines différentes se penchent ensemble sur le cas franco-ontarien, voilà qui promet beaucoup pour l'avenir.

Bien que le thème de la survivance et que la question du nationalisme soient apparus à plusieurs reprises et sous diverses formes, la plupart des communications semblaient préférer une approche moins engagée. Parmi les nombreux thèmes proposés il convient peut-être d'en signaler quelquesuns. Premièrement, plusieurs participants ont proposé l'étude des rapports entre le groupe franco-ontarien et les autres groupes ethniques (on a parlé de choc culturel, d'acculturation, de désocialisation, d'assimilation, etc.). Un autre thème repris plusieurs fois est celui du passage d'une société rurale à une société urbaine et industrielle, passage réalisé plus rapidement chez les Franco-Ontariens que chez les Québécois selon certains. Une troisième question qui apparut souvent est celle de la démographie de la population franco-ontarienne: ses origines, ses migrations, ses caractéristiques démographiques, etc. Enfin, plusieurs ont souligné l'importance d'étudier les Franco-Ontariens dans un contexte plus général: celui de l'Ontario, celui de la francophonie canadienne, celui du Canada.

Concluons en disant que ce volume rend un grand service à la recherche naissante sur l'Ontario français, et souhaitons qu'il soit le germe d'une abondante moisson d'études. 